\title{
Inflammation in the lungs of patients diagnosed with Cystic Fibrosis: Association with iron deficiency
}

\author{
Dawn Armstrong and Linsday Robinson
}

This manuscript was prepared under the supervision of Professor Lindsay Robinson, Department of Human Health and Nutritional Sciences, College of Biological Sciences.

\begin{abstract}
Cystic Fibrosis (CF) is characterized by a wide spectrum of phenotypic characteristics such as; deep coughing, increased mucous production, and weight loss. However, only recently was the role of inflammation on the etiology of the disease recognized. $\mathrm{CF}$ is characterized as a cyclic progression of infective exacerbations and stable periods initiated by the presence of Pseudomonas Aeruginosa (PA). An increase in inflammatory cytokines/mediators and a decrease in anti-inflammatory cytokines contribute to the net inflammation and overall tissue destruction of the lungs. $\mathrm{PA}$ is associated with the low iron status that is seen in $60-75 \%$ of the CF population, through the presence of iron sequestering siderophores which distract iron from the tissues. Iron deficiency (ID) initiates further symptoms such as; fatigue, tachycardia, weakness, brittle nails etc, in addition to those caused by CF. The colonization of PA may be the cause or a result of increased iron (ferritin) concentrations in the lungs, but independent of the original relationship, results in a decreased iron status. Iron is used by PA under hypoxic conditions such as in the fibrosis lung, as a source of energy. Studies on the relationship between CF and ID contribute a variety of possible causes although currently no direct connection has been discovered. At this stage, further studies in this area are needed. This review will primarily focus on the affects of CF on iron status in humans, and secondarily examine the effect of mediators of inflammation in respects to ID.
\end{abstract}

$\mathrm{C}^{\mathrm{s}}$ ystic Fibrosis (CF) is a genetic disease (autosomal recessive) that occurs in childhood and results in premature death in the early adult years. ${ }^{2,5,12,21,25,28,29}$ It is the most common lethal recessive disease and currently no cure has been discovered. ${ }^{2,14,21} \mathrm{CF}$ is a multi-system disease that presents as a spectrum of phenotypes that affect both the respiratory and gastrointestinal systems ${ }^{21}$. It can be traced to over a thousand different mutations to the cystic fibrosis transmembrane conductance regulator (CFTR). ${ }^{2}, 14,21 \mathrm{~A}$ mutation of the CFTR gene alters the function of the epithelium, which results in the production of abnormally thick mucous. The blockage of airways as a result of the mucous may result in a decrease in mobilization of iron and therefore an increase in stores. ${ }^{31}$

Even the slightest increase in iron is enough to initiate enough bacterial growth to overwhelm the immune system. ${ }^{30}$ Iron is seen increased in CF and initiates the growth and development of bacterial infections such as Pseudomonas Aeruginosa (PA). ${ }^{1,9,15,28}$ The colonization of bacteria (i.e. PA) results in recurrent episodes of chronic infection and inflammation throughout the lifespan, ${ }^{25}$ as these bacteria utilize iron sources in the lung through iron sequestering siderophores. ${ }^{1,31}$ An increase in the metabolism of iron in the epithelium results in an increased production of free hydroxyl-radicals, which damages airways, further stimulating the production of mucous, initiating a vicious cycle. $^{30}$

$\mathrm{CF}$ is an airway obstructive disorder that progresses towards the development of a hypoxic environment within the lungs. ${ }^{12}$ As a result the body initiates polycythaemia, which is an attempt to increase haemoglobin and hematocrit concentrations in the blood as a method of increasing bound $\mathrm{O}_{2}{ }^{24}$ The development of new blood cells is known as erythropoiesis and may result in ID, as it removes iron from other regions of the body. ${ }^{24}$

ID or anaemia is defined as a transferrin saturation $<16 \%$ in the blood and is commonly found in $60 \%-75 \%$ of the CF population. ${ }^{25,26,28}$ ID remains a problem even in patients that meet the recommended dietary intake (RDI) of iron, those consuming supplementation, and in those consuming a diet low in iron. ${ }^{25,}{ }^{26}$ Symptoms such as anaemia, tachycardia, fatigue, pale skin, headaches, increased work of breathing (WOB) and weakness are all characteristic of ID. ${ }^{2}$ These symptoms in addition to symptoms of $\mathrm{CF}$ leave patients in a compromised state of well being. 
Iron is found in the human body in multiple forms which include; ferritin, which is a storage protein, transferrin, an iron transport protein, and total iron, which is any unbound iron within systemic and pulmonary circulations. Both ferritin and total iron increase in patients with CF whereas transferring concentrations decrease. ${ }^{26}{ }^{31}$ Chronic inflammation is one of the first symptoms of $\mathrm{CF}$ and is the most likely to cause an increase in iron in the sputum. ${ }^{26,29,30}$ This is may be a result of the increase in mucous inhibiting any iron released from re-entering circulation, or a number of other possibilities. Although the lungs are not the only source of mucosal damage, the gastrointestinal system, specifically the pancreas, is also a common target. Blockage of the pancreas is caused by fibrosis of the organ and the production of a thick mucous, similar to that found in the lungs. ${ }^{8}$

Neutrophils are produced as an immune response to infection that increases inflammation of the target tissue. They act as the bodies' guardian by attempting to remove foreign particles. ${ }^{33}$ They release granulocytes containing sacs of enzymes that are used to digest micro-organisms (PA) retrieved through phagocytosis. Neutrophils also produce a protease known as elastase which degrades the elastin found in the airway walls, resulting in the fibrosis. ${ }^{31}$

The increase in neutrophils and macrophages results in an increased production of cytokines. ${ }^{33} \mathrm{CF}$ is associated with an increase in acute phase protein (APP) (i.e. CRP), eicosanoids (i.e. $\mathrm{LTB}_{4}$ ), pro-inflammatory cytokines (IL-6, TNF- $\alpha$ ) and decrease in anti-inflammatory cytokines (IL-6, IL-10). ${ }^{3,512,21,23,25,27,28,29} \mathrm{CF}$ patients experience a decrease in anti-inflammatory cytokine and an increase in proinflammatory cytokines, resulting in a net increase in inflammation, 5, 23, 25, 18 Down-regulation of anti-inflammatory agents results in a further increase in inflammation. $\mathrm{CF}$ is a cyclical disease: an increase in cytokines increases the production of neutrophils, and results in further damage to the system.

There are many possible causes of ID as have been discussed above. This review will focus on inflammation in the CF lung and will discuss neutrophil concentration, proinflammatory and anti-inflammatory cytokines, NO status in sputum and brochoalveolar lavage fluid (BAL) and the effects of these products on iron status in vivo.

\section{Iron Metabolism by PA in the Lungs of Patients with CF}

Iron is an essential nutrient to the growth and development of PA, the most common bacterial infection in patients with $\mathrm{CF}^{28}$ The increased colonization of PA plays both a direct and indirect role in the progressive destruction of lung tissue. ${ }^{1}$ PA normally develops in CF children around the age of 6 , and is responsible for periodic episodes of exacerbation throughout the lifespan. ${ }^{1,30,33}$ Under normal conditions the lung maintains iron levels as close to zero as possible. If iron concentrations alter from this set point there is an overwhelming response/colonization by pathogenic bacteria which use iron as energy for growth and respiration. ${ }^{30} \mathrm{PA}$ has the ability to secrete iron- binding granules known as siderophores, which increase available iron in the lungs. ${ }^{28,31}$ Patients with CF have an unusually high concentration of free iron in their lungs compared to controls, ${ }^{28,29,30}$ and this may be a result of; increased vascular permeability to the serum, increase in airway TNF- $\alpha$ (decreases iron reabsorption), airway inflammation (accumulation of iron in airways), an increase in cell death (oxidative damage), or the pseudomonas siderophores. $^{29,30,31}$

Patients with both non-exacerbated and infective exacerbated symptoms of $\mathrm{CF}$ both have an increase in available iron concentration. ${ }^{29}$ Infective exacerbated symptoms include things such as: deep coughing, increased sputum concentrations, breathlessness, fevers, weight loss, and are experienced many times during the lifespan of patients with $\mathrm{CF}^{26}$ During exacerbations patients are also found to have increased concentration of PA bacteria. ${ }^{28}$ As was stated above even the slightest increase in free iron is enough to initiate the colonization of PA. ${ }^{1,30,31}$ The increase in mucous found in CF leaves the lungs in a state of hypoxia, which means that bacteria must adapt their metabolism to anaerobic conditions. ${ }^{1,29,30}$ PA has adapted to hypoxia by developing branched aerobic respiratory side chains, termed 5-terminal-oxidases. ${ }^{1}$ For successful metabolism the PA is unique in that only one active chain is required. ${ }^{1}$ The side chains have a high affinity for $\mathrm{O}_{2}$, and allow for PA to flourish in hypoxic conditions. ${ }^{1}$ Secondarily, polycythaemia is used in the lung as a response to decrease $\mathrm{O}_{2}$ concentrations in the blood. Polycythaemia is an overproduction of red blood cells in an attempt to increase $\mathrm{O}_{2}$ delivery throughout the body. ${ }^{24}$ Patients with an infective bout of CF may have increased risk of ID due to increases in erythropoiesis. Erythropoiesis maxes out all other stores of iron available in an attempt to increase production of $\mathrm{Hb}^{24}$ However, when tested this hypothesis proved incorrect. Patients with $\mathrm{CF}$ did have a decreased $\mathrm{O}_{2}$ saturation $\left(\mathrm{SaO}_{2} \%\right)$ but haemoglobin and hematocrit values did not differ significantly from control patients. ${ }^{24}$ Therefore erythropoiesis was not an improving factor in patients with CF; an over accumulation of red blood cells did not balance hypoxemia, and cannot be the reason for a decrease in iron status in $\mathrm{CF}$.

Reid et al. collected sputum samples from patients with acute infective exacerbations, stable $\mathrm{CF}$ patients and control patients and analyzed total iron, ferritin, and tumour necrosis factor (TNF- $\alpha$ ), both prior to and following a 14 day antibiotic treatment. Patients with the infective exacerbations showed significantly higher levels of total sputum cell count (TCC), iron, ferritin, and TNF- $\alpha$ than control patients. ${ }^{29}$

A significant association was also found between concentrations of TNF- $\alpha$ and iron homeostasis. ${ }^{29}$ Sputum iron and ferritin levels, $44.4 \mu \mathrm{mol} / \mathrm{L}$ and $3.6 \mathrm{mg} / \mathrm{L}$, respectively exceeded normal ranges for iron (13-32 $\mu \mathrm{mol} / \mathrm{L})$ and ferritin $(15-300 \mu \mathrm{mol} / \mathrm{L})$. Reid also found a significant portion of the population was infected with PA, which most 
CFTR MUTATION

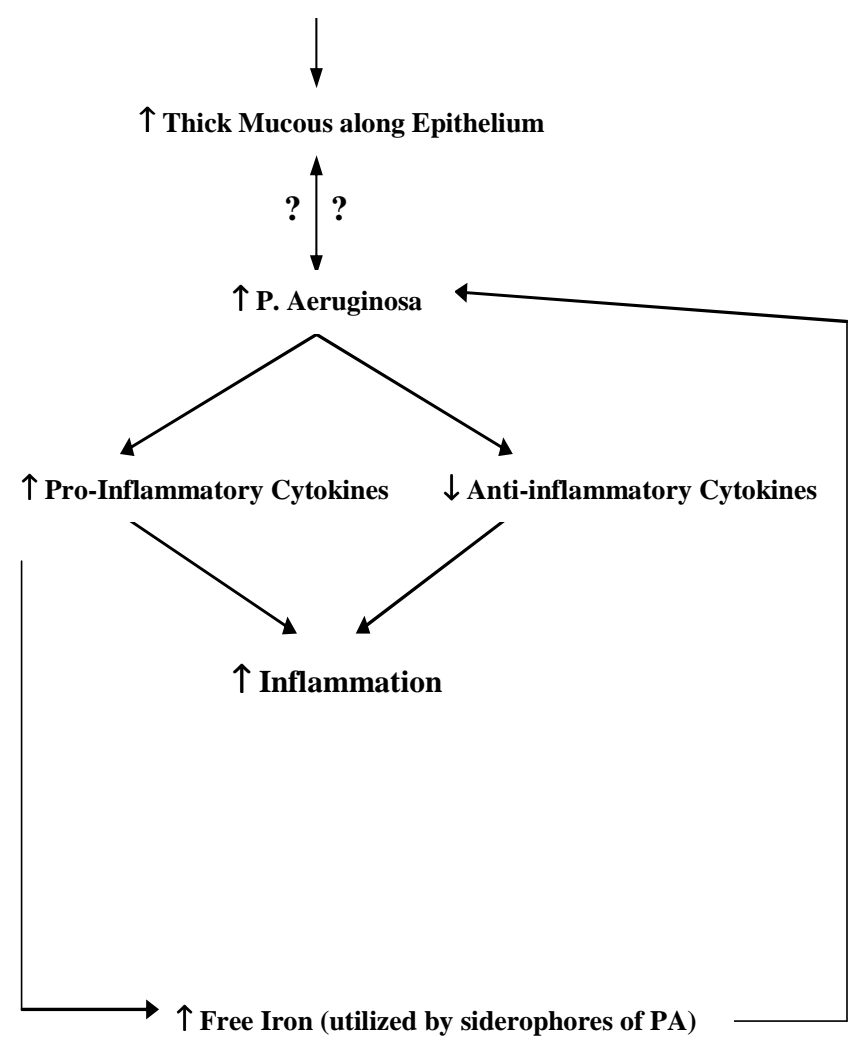

Figure 1: Possible Contributors to Iron Deficiency in CF

likely contributed to the increase in iron measured. ${ }^{29}$ This data suggests that the increase in iron may act as a fuel for PA, increase disease pathogenesis, and lead to an increased severity of respiratory failure. ${ }^{30}$ The increase in PA increases TNF- $\alpha$ released from macrophages and epithelial cells. ${ }^{30}$ The increase in PA is promoted by an increase in iron and ferritin levels in the lungs, resulting in a cyclical disease. ${ }^{29}$ Therefore research is inconclusive of whether the increase in iron seen in $\mathrm{CF}$ is an effect of increased PA, or whether the presence of PA increases available iron.

Levels of PA and iron content of the CF lung were recently found to be closely associated in CF lungs (Figure 1). ${ }^{30}$ Patients infected with PA had levels of sputum iron and ferritin of $59.73 \mu \mathrm{mol} / \mathrm{l}$ and $4002.20 \mathrm{mg} / \mathrm{L}$, respectively. Patients who were diagnosed with CF but were not infected by PA also had increased concentrations of sputum iron and ferritin when compared to a control population, $29.1 \mu \mathrm{mol} / \mathrm{l}$ and $2972.30 \mathrm{mg} / \mathrm{L}{ }^{30}$ Therefore the increase in sputum iron occurs prior to infection by PA and therefore changes in iron must predate PA (Figure 1) ${ }^{30}$ The initial increase in iron may be a result of vascular leakage due to inflammation, although the direct connection remains unknown. ${ }^{29}$ This data suggests that the initial increase in iron may be responsible for the initial colonization of PA. ${ }^{30,31}$

The main proportion of extra-cellular iron found in CF lungs is in the form of ferritin. Iron in the lungs can not be strictly supplied by damaged epithelial cells, as they contain very low concentrations of ferritin. ${ }^{31}$ It must therefore be derived from another source, which is supported by Stites et al.'s work, ${ }^{31}$ but contraindicated by research conducted by Reid et al., that suggested epithelial apoptosis was the main source of iron in the lungs. ${ }^{29}$ Iron bound to ferritin is more easily accessed then that attached to transferrin, which means that the increase in ferritin and the decrease in transferrin in the lungs influences the etiology of this disease. ${ }^{29,31}$ Reasons for the decrease in transferrin are unknown but there is reason to believe that it is cleaved by PA derived proteases. ${ }^{31}$

Ferritin is a host binding molecule that readily releases ferric $(\mathrm{Fe} 3+)$ iron in the presence of siderophores. ${ }^{15} \mathrm{PA}$ is highly effective at obtaining ferric iron from its environment and increasing its solubility. ${ }^{15,}{ }^{29}$ Therefore an increase in ferritin stores will result in an increased free iron, as a result of siderophore digestion. ${ }^{15}$ Siderophores in the sputum of patients with $\mathrm{CF}$ bind to the iron repressible outer membrane of the cell, initiating ferric ion assimilation. ${ }^{15}$ When there were increased concentrations of PA, as seen during exacerbations, there was also an increase in siderophore presence. $^{15,28}$

Increased concentrations of two specific siderophores, pyoverdine and pyochelin, were found in patients with $\mathrm{CF}^{15}$ The increase in siderophores produced by PA demonstrates the bacteria's attempt to absorb iron for survival. In summary, siderophores are scavengers of iron that incorporate proteins into the outer membranes of the bacteria, allowing for the siderophores complex to bind and absorb iron more readily. ${ }^{17}$

PA contributes both indirectly and directly to ID. The direct mechanisms involves removing iron from the airways as a source of energy through siderophore sequestering, whereas the indirect mechanism involves increasing cytokine production which, in turn increases iron storage and detracts iron from Haemoglobin $(\mathrm{Hb})$ synthesis (Figure 1). ${ }^{28}$ Previous research felt removal of PA was impossible, ${ }^{15}$ whereas recent research has used chemical and/or physical therapy to reduce exacerbation caused by $\mathrm{PA}^{29}$ Recent intervention strategies are used to treat the bacteria (the cause) instead of treating the inflammation (the effect). PA is detrimental to the body as it causes oxidative damage, inflammation, infections and uses up iron for metabolism that the body requires. ${ }^{28}$

Inflammation of the Lungs and Neutrophil Concentrations The mucous secretions of CF block the airways, resulting in not only hypoxic conditions as discussed above, but also a decreased re-absorption of iron, and endobronchial 


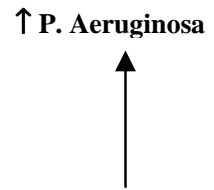

$\uparrow$ Neutrophils

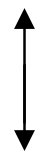

$\uparrow$ Pro-Inflammatory Cytokines

Figure 2: Relationship between P. Aeruginosa, Neutrophil Concentration and Pro-Inflammatory Cytokine Production

inflammation and/or infection. ${ }^{30,} 31$ The concentration of neutrophils in the sputum is a common marker of inflammation. ${ }^{31}$ Neutrophil concentrations are found to be significantly higher in patients with $\mathrm{CF}$ when compared with healthy subjects, $74 \%$ and $8 \%$, respectively. ${ }^{31}$ Neutrophils are an essential aspect of the autoimmune response, as they defend the body against foreign particles attempting to infect the body, however in CF, excess concentrations of neutrophils are damaging. Neutrophils fight infection by inhibiting the spread of micro-organisms through phagocytosis and by increasing the concentration of proinflammatory cytokines and proteases. ${ }^{33}$

When present in increased concentrations, neutrophils are associated with an increased production of proteases, such as elastase, which contributes to the development of fibrosis of the lungs. Proteases freely digest the elastin found in the airway walls, decreasing their elastic nature and increasing rigidity. ${ }^{19}$ The increase in elastase has secretagogue activity, which increases the production of mucous. ${ }^{19}$ The increase in PA associated with the increase in mucous, acts as a chemo-attractant to neutrophils (Figure 2). ${ }^{31}$ When neutrophils are recruited to a site of infection, the lifespan of inflammatory cytokines is increased (Figure 2). ${ }^{33}$ An increase in iron also contributes to the alterations in neutrophil behaviour. ${ }^{30}$

The severity of inflammation in the lungs is also affected by errors in apoptosis and macrophage production. Macrophages are used in the process of removing dead neutrophils from the lungs. If neutrophils have an extended lifespan due to an error in apoptosis, the result is increased recruitment of inflammatory cytokines and a decrease in recruitment of anti-inflammatory cytokines. ${ }^{33}$ Proinflammatory cytokines concentrations are increased in $\mathrm{CF}$. IL-8 is significantly higher in patients with PA $(145.5 \mathrm{ng} / \mathrm{ml})$ than in those with no bacterial content $(13.2 \mathrm{ng} / \mathrm{ml}) .{ }^{33} \mathrm{An}$ increase in IL-8 is related to an increase in neutrophil concentration and vice versa (Figure 3).

The increase in inflammation associated with $\mathrm{CF}$ is one of the main initiators of increased concentration of free and stored iron (ferritin) found in the lungs. Inflammation was found to be correlated with an increased PA population, which in turn affected iron concentrations. Inflammation independent of cause disrupts the homeostasis of iron in patients with $\mathrm{CF}^{31}$

\section{Cytokine studies in general}

Patients with CF in Nixon et al. study were compared to control patients, before and after antibiotic treatments and were found to have increased concentrations of IL- 6 , TNF- $\alpha$ and CRP. ${ }^{23}$ Therefore patients diagnosed with anaemia or iron deficiency in $\mathrm{CF}$ have an association with increased concentrations of cytokines in their sputum or BAL. Cytokine presence is one of the largest contributors to anaemia in $\mathrm{CF}$, as they cause red blood cell death and a decreased erythropoietin production. ${ }^{24}$ IL- 6 and TNF- $\alpha$ also initiate trapping of iron by macrophages, and therefore aid in the promotion of ID by inhibiting the absorption of iron at the level of the duodenum. ${ }^{12}$ A decreased absorption inhibits the production of $\mathrm{Hb}$, as it depends on iron as its central molecule. $^{12}$

Cytokines are produced from a variety of cells such as; airway, lung interstitial, air space or vascular components. ${ }^{4,23}$ They are produced in response to a variety of stimulants: hypoxia, neutrophils, increase in bacteria, and are activated by $\mathrm{T}$ and $\mathrm{B}$ lymphocytes. This occurs by lymphocytes binding foreign particles such as those that have been attacked by macrophages, and initiating further production of the $\mathrm{T}$ cells, $\mathrm{B}$ cells and cytokines. $^{23}$ The following sectionswill further examine the role of individual cytokines and related inflammatory mediators in $\mathrm{CF}$.

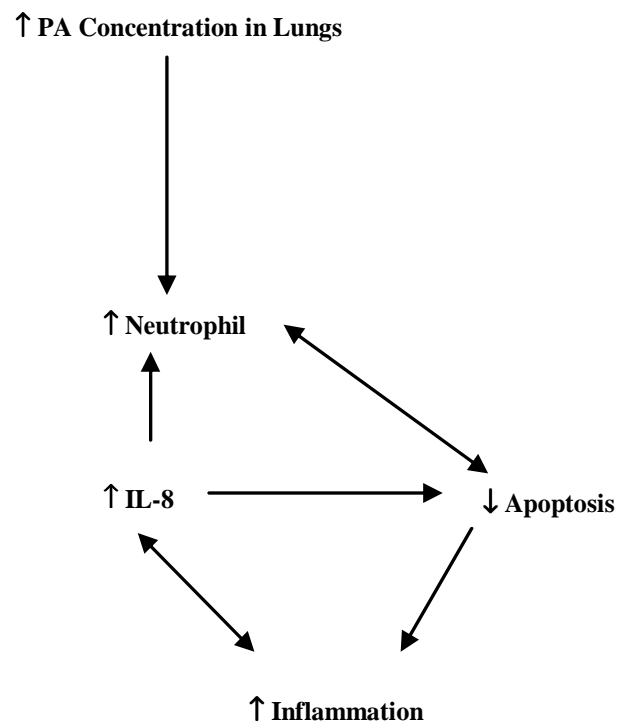

Figure 3: Cell regulation of Neutrophil Concentration an Inflammatory Cytokines in the Presence of PA in CF lungs 


\section{TNF- $\alpha$ and IL-8, Role in Respiratory Inflammation}

TNF- $\alpha$ and IL- 8 are the two major inflammatory cytokines produced by the airway epithelium and macrophages as a direct response to $\mathrm{PA}$ and indirectly to inflammation and infection $^{28,29}$ Both TNF- $\alpha$ and IL- 8 play an essential role in the over recruitment of neutrophils to damaged areas of the body. $^{20}$ The body produces TNF- $\alpha$ as a protective mechanism against PA by removing iron similar to IL-6 (as will be discussed below) however, the overwhelming concentrations result in an increase in damage to the lungs instead. $^{29}$

Concentrations of TNF- $\alpha$ were found increased in patients with CF. TNF- $\alpha$ was found at concentrations of $(448 \mathrm{pg} / \mathrm{ml})$ whereas control samples concentrations were found on average to be much lower $(9 \mathrm{pg} / \mathrm{ml}){ }^{25} \mathrm{TNF}-\alpha$ reduces the availability of iron by diverting it away from $\mathrm{Hb}$ synthesis. It also recruits iron from other areas of the body by phagocytosis, increasing available free iron in the lungs and decreasing iron with in the rest of the body. ${ }^{28}$ Reid et al found a positive correlation between a decrease in both TNF$\alpha$ and ferritin. ${ }^{28}$ ID is directly related to the severity of lung disease, and was therefore found in increased concentrations in patients with exacerbated symptoms when compared to normal patients. ${ }^{28}$

Reid et al found that an increase in TNF- $\alpha$ in the sputum of patients was closely correlated to a decrease in iron stores and an increase in sputum iron. ${ }^{27}$ TNF- $\alpha$ is responsible for up-regulation of cellular ferritin mRNA synthesis, which increases deposits of iron in the tissues of the lungs, providing a reservoir for PA. ${ }^{28}$ In support of these findings in a later study, Reid found a similar relationship, which was an increase in free iron in sputum of the lungs and an increase in TNF- $\alpha$ and IL-8. TNF- $\alpha$ was also found to correlate with IL$1 \beta .^{25}$

\section{IL-6 a Pro \& Anti Inflammatory Cytokine}

IL-6 has both pro- and anti-inflammatory functions. As an anti-inflammatory it initiates negative feedback that decreases TNF- $\alpha$ and IL- $1 \beta$ production (potent inflammatory cytokines) ${ }^{5,25}$ and plays a pro-inflammatory role through stimulation of the liver to produce Acute Phase Proteins (APP) such as $\mathrm{C}$ - reactive protein $(\mathrm{CRP}){ }^{23}$ Increased concentrations of IL- 6 are also utilized in the trapping of iron by macrophages, and play a role in the development of ID. ${ }^{12}$ Patients with CF had a higher concentration of IL-6 (8.7 +/$0.4 \mathrm{pg} / \mathrm{ml}$ ), in comparison to control patients who had significantly lower concentrations $(2.6+/-0.1 \mathrm{pg} / \mathrm{ml}){ }^{5}$ As well, patients infected with PA had higher levels $(9.3+/-$ $0.3 \mathrm{pg} / \mathrm{ml}$ ) then $\mathrm{CF}$ patients infected with another stream of bacteria $(6.9+/-0.5 \mathrm{pg} / \mathrm{ml}){ }^{5}$ However in the CF lung, IL-6 seems to play a pro-inflammatory role due to an increase in TNF- $\alpha$.

Both IL-6 and LTB $_{4}$ concentrations significantly decreased after patients with PA were treated with antibiotics for two weeks. ${ }^{5}$ Similarly, Nixon et al. found that patients with $\mathrm{CF}$ had increased levels of IL-6 of $(7.28 \mathrm{pg} / \mathrm{ml})$ compared to the control group which measured at $(0.65 \mathrm{pg} / \mathrm{ml}){ }^{23}$ After a 14 day treatment plan, patients with CF showed significant decreases in IL-6 $(2.1 \mathrm{pg} / \mathrm{ml})$ whereas the control group showed no significant changes. ${ }^{23}$ These studies both found IL-6 to play an inflammatory role. Carpagnano et al. compared a group of patients and analyzed BAL whereas Nixon et al. looked at less patients and measured concentrations in sputum. ${ }^{5}{ }^{23}$ Cytokine concen ${ }^{\mathrm{t}}$ rations present different characteristics depending on their origin in the body. More research is consequently needed in this area.

In contrast to the above studies Osika et al. found decreased IL-6 concentrations in patients with $\mathrm{CF}$ in comparison to healthy subjects and suggested that IL-6 had anti- inflammatory characteristics. ${ }^{25}$ This study measured the sputum concentration of IL-6 in a similar population to that in Carpagnano et al. and therefore is of similar quality of research. ${ }^{5}$ This study found that patients with CF had levels of IL-6 of $(26 \mathrm{pg} / \mathrm{ml})$ whereas the control group had levels of $(225 \mathrm{pg} / \mathrm{ml})$. Although Osika et al. found that there was no significant difference between $\mathrm{CF}$ patients who were infected with $\mathrm{PA},{ }^{25}$ and patients with $\mathrm{CF}$ that were not, which conflicts with results found in most other studies. IL-6 as an anti-inflammatory cytokine aids in the disruption of homeostasis found between pro and anti-inflammatory cytokines in these patients. ${ }^{25}$ Therefore the increase in inflammation contributed to by changes in IL-6 activity increase the stability of PA and therefore further increase ID.

Anaemia is a common characteristic of inflammatory diseases such as CF. Inflammation in the body results in the recruitment of a large numbers of inflammatory cytokines that mediate the immune response. ${ }^{24}$ All the factors relating to ID such as: shortened red-cell survival, a decreased erythropoietin response to anaemia and decreased erythropoietin colony formation, can be related to the presence of these substrates. ${ }^{32}$ These cytokines attempt to remove any available iron from the PA, but as a result decrease concentration throughout the body instead. What initially is meant to be a protective mechanism during acute infection consequently harms the body during chronic disease.

\section{Inflammatory Mediator; $\boldsymbol{C}$ - Reactive Protein}

CRP is an APP that increases proportionally with IL-6. Increased concentrations of IL- 6 stimulate the production of CRP in the liver. ${ }^{23}$ Inflammation initiates an increase in recruitment of IL-6, which results in increased CRP released in the serum and sputum. ${ }^{27}$ Mechanistically CRP binds to foreign or damaged particles and removes them from the body by actively binding and promoting the activity of macrophages. $^{27}$ CRP aids in the development of ID by inducing the trapping of iron in macrophages, and decreasing its absorption as it stimulates macrophage activity. CRP also initiates cytokine induced hepatic expression of hepcidin, 
which is produced in the gastrointestinal tract and inhibits the absorption of iron in the duodenum. ${ }^{12}$ The highest concentrations of CRP were recorded during exacerbation, but after 14 days of antibiotic treatment CRP levels were found to level off. ${ }^{27}$ Increased values of CRP result in a decrease in available iron. ${ }^{12}$

Fischer et al. (2007) found that $40 \%$ of CF patients presented with high levels of CRP, $(0.5 \mathrm{mg} / \mathrm{dl})$ compared with normal values of $(<0.22 \mathrm{mg} / \mathrm{dl})$. After 3 and 6 months $60 \%$ and $67 \%$ of the group, respectively, had an increase in CRP. These patients had two times the concentration of CRP than the control patients. $88 \%$ of the patients which had presented originally with non-exacerbated symptoms experienced an exacerbated infection as a result of PA by the end of the 12-month study, ${ }^{12}$ which also demonstrates the regular occurrence of exacerbations throughout the lifespan. As a result, a correlation is seen between CRP and infection. During periods of infection CRP levels were higher then in the non-exacerbated CF lung. ${ }^{12}$

Nixon et al. found that CRP levels within each individual showed no increase over the 14 day study although consistent with Fischer et al. CRP values were greater in patients with $\mathrm{CF}$ than in the control group. ${ }^{23,12}$ Both Nixon et al. and Fischer et al. found that once CRP levels reach their maximum, an increase in infection is no longer proportional to CRP. ${ }^{23,12}$ Exacerbated CF patients began the trial with CRP levels of $21.32 \mathrm{ug} / \mathrm{ml}$ but after treatment showed a significant decrease to $2.75 \mathrm{ug} / \mathrm{ml}$ whereas before and after treatment control patients showed no significant change $0.33 \mu \mathrm{g} / \mathrm{ml}$ to $0.34 \mu \mathrm{g} / \mathrm{ml} .^{23}$

\section{IL-10, an Anti-inflammatory Cytokine}

The airways of patients affected with CF are relatively deficient in the anti-inflammatory cytokine IL-10. 3, 18, 25 IL10 was recorded at $(24 \mathrm{pg} / \mathrm{ml})$ in patients with $\mathrm{CF}$ whereas control subjects recorded a significantly lower average concentration of $(45 \mathrm{pg} / \mathrm{ml}){ }^{25}$ Sputum measurements in the control population were induced by treating subjects by induction of $200 \mu \mathrm{g}$ of inhaled salbutamol, as sputum is not produced under healthy conditions. ${ }^{25}$ IL-10 is an inhibitory cytokine that acts on IL-8 and TNF- $\alpha$ (pro-inflammatory). Unlike CRP which decreased when exacerbation was complete, IL-10 levels remain low in all stages of illness. Therefore low concentrations of IL-10 allow for the exaggerated effect of inflammatory cytokines IL-8 and TNF$\alpha$ and indirectly contribute to ID and an increase inflammatory damage to the lung. ${ }^{3,18}$

\section{Nitric Oxide a Marker of Airway Inflammation}

Over the last ten years a number of studies have focused on nitric oxide (NO) and its role in pulmonary disease. NO is a marker of airway inflammation and is found in the EBC of individuals with chronic respiratory disease. ${ }^{13} \mathrm{NO}$ is released from activated macrophages, neutrophils and infected lung epithelial cells, by stimulation of $\mathrm{T}$ and $\mathrm{B}$ immune cells. ${ }^{16}$
NOS, is the enzyme that is responsible for the proliferation of NO and is positively influenced by the presence of PA, and TNF- $\alpha$, which are seen increased in CF. ${ }^{13,16}$ Therefore, in individuals with increased NO, an increase in NOS can be assumed. ${ }^{13}$ Nitrite $\left(\mathrm{NO}_{2}{ }^{-}\right)$and Nitrate $\left(\mathrm{NO}_{3}{ }^{-}\right)$are the two stable end products produced by $\mathrm{NO}$ metabolism and are found in increased concentrations in $\mathrm{CF} .{ }^{16} \mathrm{NO} / \mathrm{NOS}$ like other inflammatory factors contributes to ID through activating an increase in neutrophils, macrophages and damaged epithelial cells. ${ }^{13,26}$ As a response to the infiltration of bacterial cultures, the body responds by increasing NO. ${ }^{26}$ Again, CF is a cyclic disease, and an increase in inflammation will further increase concentrations of PA resulting in increased utilization of iron, contributing to the overall ID in CF patients.

Patients with CF unexpectedly present with low levels of NO and high levels of NOS. 1, 13, 16, 26 Ho et al. hypothesized that due to the increase in mucous in the airways, NO is trapped close to the aqueous membrane, which allows for complete metabolism into $\mathrm{NO}_{2}^{-}$and $\mathrm{NO}_{3}{ }^{-16}$ When $\mathrm{NO}$ is trapped neighbouring the aqueous membrane of the epithelial cells, a reaction occurs with reactive oxygen species or with water and oxygen, stabilizing the molecule. Ho et al. found that $\mathrm{NO}_{2}{ }^{-}$levels were significantly higher in patients with $\mathrm{CF}$ $(2.15 \mu \mathrm{m}$ versus control $0.36 \mu \mathrm{m})$ whereas NO showed no significant difference between the two populations (3.8 versus control $5.2 \mathrm{ppm}) .{ }^{16}$ Nitrate levels which are much lower in $\mathrm{CF}$, maybe due to its possible metabolic role in PA respiration. ${ }^{1}$ Under hypoxic conditions, nitrate may be used as a substitute of oxygen. ${ }^{1}$ It is therefore most likely that $\mathrm{CF}$ results in an increased production of NO but most of it is converted to bi-products. ${ }^{16,22}$ The exact reasoning remains unknown as to whether it is an increase in NO metabolites which there is research to support or a decrease in the production of NO. ${ }^{13}$

Bacteria which utilize iron as a source of energy may be responsible for the decrease seen in NO and hence the abnormal symptoms that are seen in CF. Consequently, the greater the inflammation, the more PA that can thrive on available iron and the larger the amount of $\mathrm{NO}$ that is metabolized by the PA to $\mathrm{NO}_{2}^{-}$. Possible research may be focused on developing a correlation between concentrations of $\mathrm{NO}_{2}{ }^{-}$and a decrease in iron levels in the blood.

\section{Iron Deficiency in the Gastro intestinal (GI) tract}

Although this paper focuses on the decrease in iron at the respiratory level it will briefly touch on the effects of ID in the GI system. Davis \& Biggs' paper, which is original literature on the relationship between the pancreas and its role in iron absorption, found that a decrease in the function of the pancreas causes a decrease in iron absorption. ${ }^{8}$ Davis \& Biggs' study, however, was performed on rats, ${ }^{8}$ which must be considered when applying the findings to humans. When ferrous sulphate $\left(\mathrm{FeSO}_{4}\right)$ was injected into the isolated and perfused region of the rat's small intestine ( $\mathrm{pH}$ of 1 ), the 
mean value of iron absorption was $51 \%$, whereas when the same dosage of iron was given with pancreatic extract, iron absorption decreased to $29 \%{ }^{8}$ It was concluded that pancreatic secretions play some role in the absorption of iron in the jejunum, and are associated with chronic $\mathrm{CF}$, however, it is important to note that pancreatic secretions are only produced in $\mathrm{CF}$ once severe pancreatic damage has occurred. The natural $\mathrm{pH}$ of the jejunum in rats is roughly $6.4^{7}$ whereas a human's natural intestinal $\mathrm{pH}$ ranges between $6.63-7.4,{ }^{11}, 34$ which means that the slight differences between the data sets must be considered, and must give way to confirmation by further studies performed on human subjects (in vivo). In conjunction with these findings, research by Pond et al. also found that iron absorption is inhibited by an increase in volume of mucous on the pancreas. ${ }^{26}$ When pancreatic enzymes are inhibited, a decrease in iron absorption can be observed, eventually leading to ID, which can also be observed. ${ }^{26}$ For this reason, pancreatic extract, as a result of mucous production, resulting in a decreased absorption of iron, has been suggested to play an inhibitory role.

Recently, Fischer et al. tested patients for exocrine pancreatic insufficiency and found that all patients were limited in their ability to secrete pancreatic enzymes. ${ }^{12}$ Problems with this study included set backs such as looking at a small number of patients (at no fault to the researcher as CF patients are hard to find for testing), as well as using single measurements upon which to base comparisons, as these, in combination, may have been inaccurate when assessing overall health of subjects. ${ }^{12}$ This study also found that even patients who were taking iron supplementation prior to the study had iron levels below cut off. ${ }^{12}$ These findings suggested that ID is caused by an inability to absorb nutrients, and is also not the result of a dietary deficiency. Recent research, in contrast, has found evidence to support the hypothesis that that the degree of ID may be unrelated to pancreatic insufficiency, although further research is needed into this area. ${ }^{28}$ Reid et al. found that even when patients were given pancreatic supplements, iron levels showed no improvement. ${ }^{28}$ Absolute reasons for these observations have yet to be discovered and may also have some relation to other GI factors.

\section{CONClusion AND Future ReSEARCH}

Individuals with $\mathrm{CF}$ present a multitude of phenotypic mutations, along with significant sources of illness, which are together associated with an increase in mucous excretion by the lungs and pancreas. The increased mucous results primarily in a blockage of airways, producing a hypoxic environment, and results secondarily in an increase in inflammation. Research is unclear as to whether inflammation is the source of the colonization of PA, or whether PA is the reason behind the body's inflammatory response. Independent of the cause, both inflammation and $\mathrm{PA}$ result in a decrease in iron and ferritin in both the blood and throughout the body. One of the areas of future research should be finding whether a direct connection between the role of NO, as an inflammatory marker, and ID is present. As well, further studies geared to adjusting the balance between the increase in pro-inflammatory, and decrease in antiinflammatory cytokines and mediators, are required to ultimately improve health and wellness within patients. Research should emphasize the importance of treating PA and the sources of inflammation, as opposed to treating symptoms, not only to increase patients' lifespan, but more importantly, to enrich their quality of life.

\section{REFERENCES}

1. Alvarez-Ortega C, Harwood CS. Responses of Pseudomonas Aeruginosa to low oxygen indicate that growth in the cystic fibrosis lung is by aerobic respiration. Molecular Microbiology 2007; 65: 153-165

2. Ater JL, Herbst JJ, Landaw SA, O'Brien RT. Relative Anaemia and iron deficiency in cystic fibrosis. Pediatrics 1983; 71:810-814

3. Bonfield TL, Konstan MW, Berger M. Altered respiratory epithelial cell cytokine production in cystic fibrosis. J. Allergy Clin Immunol 1999; 104: 72-78

4. Bonfield TL, Konstan MW, Burfeind P, Panuska JR, Hilliard JB, Berger M. Normal bronchial epithelial cells constitutively produce the anti-inflammatory cytokine IL-10 which is down-regulated in cystic fibrosis. American Journal Respiratory Cellular Molecular Biology 1999; 13: 257-26

5. Carpagnano GE, Barnes PJ, Geddes DM, Hodson ME, Kharitonov SA. Increased leukotriene $\mathrm{B}_{4}$ and interleukin-6 in exhaled breath condensate in cystic fibrosis. Am J Respir Crit Care Med 2002; 167: 11091112

6. Courtney JM, Ennis M, Elborn JS. Review Article: Cytokines and inflammatory mediators in cystic fibrosis. Journal of Cystic Fibrosis 2004; 3: 223-231

7. Crouthamel WG, Abolin CR, Hsieh CR, Lim JK. Intestinal $\mathrm{pH}$ as a factor in selection of animal models for bioavailability testing. Journal of Pharmaceutical Sciences 1975; 64: 1726-1727

8. Davis AE, Biggs JC. The pancreas and iron absorption. Gut 1965; 6: 140-142

9. Davis P. Clinical pathophysiology and manifestations of lung disease. In: Yankaskas J, Knowles M, editors. Cystic Fibrosis in Adults. Philadelphia: LippincottRaven Publishers; 1999:45-67

10. Doring G. Cystic Fibrosis respiratory infections: interactions between bacteria and host defence. Monaldi Arch Chest Dis 1997; 52: 363-366 
11. Evans DF, Pye G, Bramely R, Clark AG, Dyson TJ, Hardcastle JD. Measurement of gastrointestinal $\mathrm{pH}$ profiles in normal ambulant human subjects. Gut 1988; 29: 1035- 1041

12. Fischer R, Simmerlein R, Huber RM, Schiffl H, Lang SM. Lung disease severity, chronic inflammation, iron deficiency, and erythropoietin Response in Adults with cystic fibrosis. Pediatric Pulmunology 2007; 42: 1193-1197

13. Grasseman H, Ioannidis I, Tomkiewicz RP, Groot HD, Rubin BK, Ratjen F. Nitric oxide metabolites in cystic fibrosis lung disease. Arch Dis Child 1998; 78: 49-53

14. Gurwitz P, Corey M, Francis P. Perspectives in Cystic Fibrosis, Pediatric Clinic.1979; 26: 603

15. Haas B, Kraut J, Marks J, Zanker SC, Castignetti D. Siderophore presence in sputa of cystic fibrosis patients. Infection and immunity 1991; 59: 3997-4000

16. Ho LP, Innes JA, Greening AP. Nitrite levels in breath condensate of patients with cystic fibrosis is elevated in contrast to exhaled nitric oxide. Thorax 1998; 53: 680684

17. Konisky J. Bacterial Outer Membranes Biogenesis and Functions (Inouye, M.Ed.) 1979; 319-359

18. Konstan MW, Berger M. Infection and inflammation in the lung in cystic fibrosis. In: Davis PB, ed. Cystic Fibrosis. New York: Marcel Dekker 1995; 219-276.

19. Konstan MW, Berger M. Current Understanding of the inflammatory process in cystic fibrosis: onset and etiology. Pediatric Pulmonology 1997; 24: 137-142

20. Kurlandsky LE, Bennink MR, Webb PM, Ulrich PJ, Baer LJ. The absorption and effect of dietary supplementation with omega-3 fatty acids on serum Leukotriene $\mathrm{B}_{4}$ in patients with cystic fibrosis. Pediatric Pulmunology 1994; 18: 211-217

21. Levy H, Kalish LA, Huntington I, Weller N, Gerard C, Silverman EK, Celedon JC, Pier GB, Weiss ST. Inflammatory markers of lung disease in adult patients with cystic fibrosis. Pediatric Pulmunology 2007; 42: 256-262

22. Marteus H, Tornberg DC, Weitzberg E, Schedin U, Alving K. Origin of nitrite and nitrate in nasal and exhaled breath condensate and relation to nitric oxide formation. Thorax 2004; 60: 219-225
23. Nixon LS, Yung B, Bell SC, Elborn JS, Shale DJ. Circulating immunoreactive interleukin-6 in cystic fibrosis. Am J Respir Crit Care Med 1998; 157: 17641769

24. O'Connor TM, McGrath DS, Short C, O'Donnell MJ, Sheehy M, Bredin CP. Cubclinical anaemia of chronic disease in adult patients with cystic fibrosis. Journal of Cystic Fibrosis 2001; 1: 31-34

25. Osika E, Cavaillon JM, Chadelat K, Boule M, Fitting C, Tournier G, Clement A. Distinct sputum cytokine profiles in cystic fibrosis and other chronic inflammatory airway disease. Eur Respir J 1999; 14: 339-346

26. Pond MN, Morton AM, Conway SP. Functional iron deficiency in adults with cystic fibrosis. Respiratory Medicine 1996; 90: 409-413

27. Rayner RJ, Wiseman MS, Cordon SM, Norman D, Hiller EJ, Shalet DJ. Inflammatory markers in cystic fibrosis. Respiratory Medicine 1991; 85: 139-145

28. Reid DW, Withers NJ, Francis L, Wilson JW, Kotsimbos TC. Iron deficiency in cystic fibrosis: Relationship to lung disease severity and chronic pseudomonas aeruginosa infection. Chest 2002; 121: 4854

29. Reid DW, Lam QT, Schneider H, Walters EH. Airway iron and iron-regulatory cytokines in cystic fibrosis. Eur Respir J 2004; 24: 286-291

30. Reid DW, Carroll V, O’May C, Champion A, Kirov SM. Increased airway iron as a potential factor in the persistence of Pseudomonas Aeruginosa infection in cystic fibrosis. Eur Respir J 2007; 30: 286-292

31. Stites SW, Walters B, O'Brien-Ladner AR, Bailey K, Wesselius LJ. Increased iron and ferritin content of sputum from patients with cystic fibrosis or chronic bronchitis. Chest 1998; 114: 814-819

32. Vernet $M$. The transferrin receptor: its role in iron metabolism and its diagnostic utility. Ann Biol Clin 1999; 57:9-18

33. Watt AP, Courtney J, Moore J, Ennis M, Elborn JS. Neutrophil cell death, activation and bacterial infection in cystic fibrosis. Thorax 2005; 60: 659-664

34. West JB, 2005, Respiratory Physiology: The Essentials: Seventh Edition, United States of America, Lippincott Williams \& Wilkins 